\title{
A demonstration of the costs and benefits of expertise in recognition memory
}

\author{
HAL R. ARKES and MARILYN R. FREEDMAN \\ Ohio University, Athens, Ohio
}

\begin{abstract}
In their area of expertise, experts know more potentially interfering facts than nonexperts do, yet their memory is superior to that of nonexperts. This has been termed the "paradox of interference." We proposed that the ability of experts to go beyond the information given allows them to infer the presence of items that might not otherwise be remembered. However, such inferences can also be detrimental to accurate recognition memory in that such inferences might become confused with actual targets. We examined the benefits and costs of expertise in two recognition memory experiments in which experts and nonexperts participated. Experts in Experiment 1 were knowledgeable about baseball; those in Experiment 2 were knowledgeable about Ohio geography. Distractors in both studies bore a synonymous, an inferential, or no special relation to the targets. In the last instance, experts had recognition memory superior to that of the nonexperts. When the distractors were related to the targets, however, the nonexperts were superior. We proposed that experts' inferential behavior is a contributor to their generally superior memory, but that task demands can convert this asset into a liability.
\end{abstract}

Smith, Adams, and Schorr (1978) pointed out that many theories of memory posit interference between related facts at the time of retrieval. According to these theories, since experts know an enormous number of facts about a topic, they should suffer from substantial interference and thus exhibit poor memory. On the contrary, within their area of expertise, experts appear to retrieve an astonishing number of facts with ease (deGroot, 1965). Smith et al. (1978) referred to this contradiction as the "paradox of interference." In the present paper, we attempt to resolve this paradox by demonstrating that although expertise in a subject area is often advantageous to memory in that area, it can indeed be disadvantageous under certain circumstances.

First, we need to examine why related facts are thought to foster interference. Investigators within the classical interference theory tradition (e.g., Martin, 1965; Osgood, 1949) have suggested that similarity of responses has a substantial effect on memory. When a person tries to retrieve an item from memory, facts related to the target item are often confused with it, while dissimilar facts are not. Thus, under many circumstances, interference is increased and memory is decreased when similar responses are present.

The more recent theories of Anderson (1976) and Anderson and Bower (1973) have some features that are similar to those of classical interference theory. For example, Anderson and Bower stated that if a node (such as a person's name) has multiple predicates attached to it, then each predicate must be tested sequentially

The authors' mailing address is: Department of Psychology, Ohio University, Athens, Ohio 45701. in order to determine which is the proper one to be recalled. If only one predicate is attached to the node, then the process is much simpler, of course. Therefore, as in classical interference theory, these more recent information-processing theories also predict that related facts foster output interference.

Smith et al. (1978) suggested that by integrating closely associated facts into a single theme, experts are able to reduce interference dramatically. To use one of the Smith et al. examples, suppose a person was presented with "Marty broke the bottle" and "Marty did not delay the trip" and that these two facts were later integrated by the presentation of a third sentence, "Marty christened the ship." Subjects who learned this integrating statement were asked to verify one of the three statements in a subsequent reaction time task. Smith et al. found that these subjects could verify one of three integrated facts about as quickly as other subjects could verify the two original unintegrated facts.

One of the explanations offered by Smith et al. (1978) to account for the benefit of integration was that "many script facts are accessed as a unit," and therefore "the number of ... facts should have little influence on access time" (p. 461). Thus, when a subject hears a sentence that informs him or her that a ship-christening scenario is being described, the three related sentences become amalgamated into that script. Subjects could subsequently access this single unit more quickly than they could access the unintegrated facts.

However, Reder and Anderson (1980) suggested that this cognitive economy occurred because integration subjects merely verified whether a test sentence such as "Marty broke the bottle" was consistent with a shipchristening scenario. The subjects did not bother to 
retrieve any of the initially presented sentences to verify whether the test sentence actually matched one of them. To support their viewpoint, Reder and Anderson showed that when thematically related distractors were used in the verification tests (e.g., "Marty was sprayed with champagne"), the benefit of integration was eliminated: Three integrated facts led to longer verification times than did two facts. Reder and Anderson asserted that the economy of integration was lost in this instance, because with thematically related foils, integration subjects had to retrieve each fact in order to verify it rather than merely making a consistency judgment. Thus, Reder and Anderson (1980) showed that this benefit of expertise-integration of facts into a theme-may be limited to certain types of memory situations.

Arkes and Harkness (1980) showed that there are costs of expertise. Subjects in one of their studies were students in training to become hearing and speech therapists. Subjects were first shown a list of symptoms characteristic of Down's syndrome. Those who were able to make the diagnosis of Down's syndrome later incorrectly "remembered" Down's syndrome symptoms that had not been presented. Being expert enough to discern the correct diagnosis was a detriment to accurate symptom recognition.

In a related study, Sulin and Dooling (1974) presented subjects with sentences that constituted a story about a person. Correct rejection of thematically related foils was high after 1 week, unless subjects were told that the person was someone famous, for example, Hitler, and the foils contained information closely associated with that person, for example, hated Jews. In that case, subjects incorrectly thought that those foils had been presented a week earlier. Thus, when subjects were able to use their knowledge about a historical figure, they did worse than when this knowledge was irrelevant to the task. One reason that knowledgeable people may actually be at a disadvantage in some of these memory tasks is that they, more than novices, are able to make certain inferences. When asked if he left a tip at the restaurant last Tuesday, the regular diner might infer that he had done so even if he had not. His restaurant script (Schank \& Abelson, 1977) would not include this "slot." A visiting hermit, when asked the same question, would reply more accurately. Never having been to a restaurant before last Tuesday, he would not answer "yes" by default if the memory for the tip were weak. By being unable to make an inference about what he likely did at the end of the meal. the hermit would achieve more accurate recall on this item. Note that the "expert" would be penalized for his or her prior knowledge. Yet Charness (1979), Chiesi, Spilich, and Voss (1979), Egan and Schwartz (1979), and Reitman (1976) have shown that experts have far better memory than do nonexperts in the appropriate domain of expertise. Furthermore, Reder and Ross (1983) showed that, compared with those who know few facts about a concept, those who know more facts show faster recognition times to these facts under certain circumstances. Can knowledge be both an asset and a liability?

In the present studies, we attempted to demonstrate both the costs and the benefits of expertise. In so doing, we propose a resolution to the paradox of interference. Unlike the Reder and Anderson (1980) and Smith et al. (1978) studies, we used people who actually differed in their real-world knowledge. In Experiment 1, we contrasted experts and novices in the domain of baseball knowledge. In Experiment 2, we contrasted groups with differing geographical knowledge of central and southeastern Ohio.

\section{EXPERIMENT 1}

Chiesi et al. (1979) found that those with a great deal of knowledge about baseball had superior recognition for baseball-related information compared with those with less baseball knowledge. This is a typical benefit of expertise. By introducing appropriate distractors into the recognition test in a manner similar to that of Reder and Anderson (1980), we showed that expertise has its costs.

\section{Method}

Subjects. Thirty-two undergraduates received course credit for their participation in the experiment.

Stimulus materials. Four baseball stories were used in this study. Each story contained two targets for the subsequent recognition test. One of these targets bore either a synonymous relation to its distractor (two stories) or an inferential relation to its distractor (two stories). For example, one synonym pair contained the following target: "The Cubs' first and third basemen crept in close expecting a sacrifice." The distractor sentence was identical except that the final word was changed to "bunt." "Sacrifice" and "bunt" are extremely similar in meaning, a similarity we assumed would be noticed only by those with high baseball knowledge. The other synonym target-distractor pair differed only in the substitution of the words "off-speed pitch" for "change-up."

An example of an inferential target was, "The runner from third scored." This sentence occurred near the end of a story in which a ground ball was hit to an infielder with men on first and third with one out. The story also contained the information that the team in the field attempted a double play. Since readers of the scenario were told that the runner on third scored, knowledgeable readers could have inferred that the attempt at a double play had failed. The distractor was "The batter was safe at first," corresponding to the inference we believed that only the baseball "experts" would make.

The second target in each story was the "control" target. It was paired with a distractor with which it bore neither a synonymous nor an inferential relation. For example, "Bench moved up to second base" was one target; "Bench moved up to third base" was the corresponding distractor.

The stories ranged in word length from 160 to 178 . They ranged in readability (Flesch, 1948) from 74.4 to 80.0 .

We should point out that one type of counterbalancing was not possible in this study. The targets could not serve as distractors for half of the subjects in each group, and the distractors could not serve as targets for half of the subjects in each group. The inference vignettes were written such that the experts presumably would generate a logical conclusion. This conclusion had to be a distractor. It could not be a target unless the vignette were rewritten so as to generate a completely new 
situation with a completely new conclusion-distractor. We would not know if these two vignettes and their conclusion-distractors were comparable in a number of ways. (The same problem existed for all control statements also.) To avoid this problem, we kept the same sentences as targets and distractors for everyone.

Procedure. The subjects were tested in six groups of 25 to 40 students each. The experimenter began by telling the subjects that they would be asked to read and then to remember information about four baseball stories. The experimenter then explained that, later in the hour, a 1-to-7 rating scale would be used to test recognition memory, where 1 meant that "you're absolutely positive" that a sentence was in one of the four baseball stories and 7 meant the opposite. After this scale had been explained fully, the stories were handed out to all subjects. Each story was on a separate sheet with the title at the top. The order of the stories was randomized. The subjects were given $1 \mathrm{~min}$ to read each story.

The stories were then collected while a baseball quiz was being given out. This 45 -item quiz was based on one used by Chiesi et al. (1979). After $20 \mathrm{~min}$, the baseball quizzes were collected, and a 10-min arithmetic task was given out. At the conclusion of that task, recognition memory answer sheets were given out.

At the top of these sheets was a restatement of the 1-to-7 rating scale. The experimenter emphasized that the subjects should rate whether each exact sentence was seen in one of the four stories. Sixteen slides were presented for $8 \mathrm{sec}$ each. Each slide contained a story title at the top and a sentence in the middle. The order of the slides was carefully counterbalanced: For half of the synonym, inference, and control pairs, the distractor occurred first; for the other half, the target occurred first. For three of the six groups of subjects, the slides were shown in the reverse order. This had the effect, for these subjects, of making the target occur first for the target-distractor pairs on which the forward-order subjects had seen the distractor first, and conversely. There was always an interval of eight slides between the two members of each target-distractor pair.

\section{Results}

The top 16 scorers on the baseball quiz were deemed the "experts." Their average number of questions answered correctly was 37.0 (range $=33-42$ ). The bottom 16 scorers, the "nonexperts," averaged only 3.1 questions correct (range $=1-5$ ). ${ }^{1}$

We performed a 2 (slide order) $\times 2$ (baseball knowledge) $\mathrm{x} 3$ (sentence type: control, synonym, inference) $\mathrm{x}$ 2 (story: number 1 , number 2 ) $\times 2$ (target-distractor) analysis of variance on the recognition ratings with repeated measures on the last three factors. ${ }^{2}$ The alpha level was set at .025 . The slide-order and story-number variables were merely nuisance factors.

The predicted knowledge $\mathrm{x}$ sentence type $\mathrm{x}$ targetdistractor interaction was significant $[\mathrm{F}(2,56)=7.82$, MSe $=4.61]$. This indicated that those with differing levels of baseball expertise rated the targets and distractors differently, depending on the story type. Although this was the interaction of central interest, we also noted a significant knowledge $x$ sentence type $x$ story interaction $[\mathrm{F}(2,56)=10.18, \mathrm{MSe}=2.54]$. Inspection of these means led us to the discovery that we had made a mistake in the construction of the inference slides. This error resulted in a serendipitous and instructive finding, which will be discussed when the inference data are analyzed separately.

First, to elucidate the central three-way interaction, we examined the control, synonym, and inference sentences separately. The left panel of Figure 1 depicts the anticipated result for the control sentences: Highknowledge subjects were more likely than low-knowledge subjects to judge the target "old" and the distractor "new." This is the typical benefit of expertise. These control-sentence recognition ratings were analyzed by a 2 (knowledge) $\times 2$ (Story 1 or Story 2 ) $\times 2$ (targetdistractor) analysis of variance. ${ }^{3}$ Baseball knowledge was significant $[\mathrm{F}(1,30)=6.09, \mathrm{MSe}=2.89]$, as was targetdistractor $[\mathrm{F}(1,30)=44.42, \mathrm{MSe}=2.27]$. These were qualified by the predicted knowledge $\mathrm{x}$ target-distractor interaction $[\mathrm{F}(1,30)=10.24, \mathrm{MSe}=2.26]$.

Second, we examined the synonym-sentences' recognition ratings separately. For synonym sentences, we were really testing whether the experts and nonexperts differed in their verbatim memory, since synonym targets and distractors have nearly identical meanings, of course. Reference to the middle panel of Figure 1 reveals that, contrary to the prior analysis, the highknowledge subjects were less likely than the low-knowledge subjects to judge the targets "old" and the distractors "new." This is a cost of expertise.

These synonym recognition ratings were analyzed by a 2 (knowledge) $\times 2$ (Story 1 or Story 2 ) $\times 2$ (order) $\mathrm{x} 2$ (target-distractor) analysis of variance. As was the case in the prior analysis, the knowledge $x$ targetdistractor interaction was significant $[F(1,28)=11.20$, $\mathrm{MSe}=4.35] .^{4}$

The analysis of the inference sentences was complicated by the fact that, due to an error, the two inference stories were not comparably tested. We correctly tested Story 1, which we presented above as an example: high-knowledge people would assume that if a runner scored from third on an attempted double play with men on first and third, then the runner on first must have been safe. Therefore, we predicted that highknowledge people would be less likely than low-knowledge people to correctly reject the distractor "The

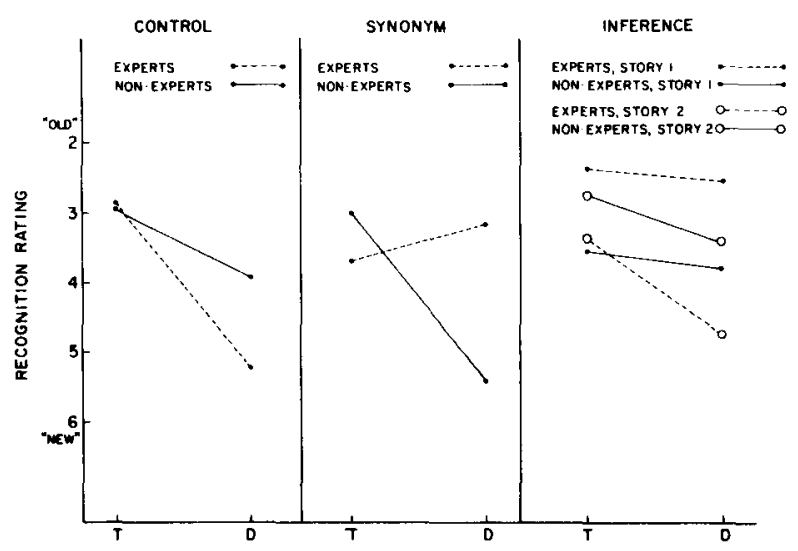

Figure 1. Left: Recognition of control sentences by experts and nonexperts. Center: Recognition of synonym sentences. Right: Recognition of inference sentences with the sentences from the two stories graphed separately. 
runner was safe at first." Because we thought that highknowledge people would have inferred this statement, they might falsely recognize it as being "old." Indeed, the experts called the distractor "old" to a significantly greater extent than the novices did $(2.6$ to 3.8$)[\mathrm{t}(30)=$ 2.85]. This is a cost of expertise.

The other inference story dealt with a manager's bringing in a new pitcher to face a left-handed power hitter. The target sentence was "The new pitcher came into the game for the Red Sox." Baseball experts know that managers typically bring in left-handed pitchers to face left-handed batters. Therefore, when experts saw the distractor "The right-hander came into the game for the Red Sox," they knew that this distractor was most unlikely. Therefore, unlike the situation in the prior inference story, their high knowledge should help the experts on the sentence-recognition task by enabling them to reject the distractor rather confidently. In fact, the experts called this distractor "new" to a significantly greater extent than the novices did (4.7 to 3.4$)[\mathrm{t}(30)=$ 3.01]. This is a benefit of expertise. The data for both inference stories are depicted in Figure 1c.

\section{Discussion}

The results show that expertise can be an asset or a liability. Experts had superior recognition on the control sentences, an expected result found earlier by Chiesi et al. (1979). On the synonym sentences, however, the experts did significantly more poorly than did the novices. This also occurred in the inference story in which the distractor contained a fact that could be inferred from the story. The experts demonstrated superiority, however, when the distractor contained a fact whose opposite could be inferred from the story.

A number of other studies have shown that inferred items are often falsely remembered to have been presented. (See Harris, 1981, for a summary of such studies.) For example, Bransford, Barclay, and Franks (1972) had subjects listen to the following sentence: "Three turtles rested on a floating $\log$, and a fish swam beneath them." These subjects later falsely asserted they had heard "Three turtles rested on a floating log, and a fish swam beneath it." Since the second sentence can readily be inferred from the first one, subjects became confused as to which one was actually presented.

Our first study differed from the Bransford et al. (1972) research in two important ways. First, we showed the importance of expertise. Second, we suggest that the relation between our inferred and presented sentences is far more subtle than the relation between inferred and presented sentences in prior research. No sentence even remotely similar to the "The runner was safe at first" was included in the story. Yet this distractor sentence was given an even "older" rating by the experts than the nonexperts gave the target.

We examined this process further in Experiment 2. We changed the subject matter from knowledge about baseball to knowledge about Ohio University and the nearby area of Ohio.

\section{EXPERIMENT 2}

\section{Method}

Subjects. Thirty-two undergraduates participated in the experiment. Sixteen attended Ohio University; 16 attended the University of Oregon. The first group was deemed the Ohio cxperts.

Stimulus materials. The four stories used in this study varied in word length from 150 to 164 and in readability (Flesch, 1948) from 71.5 to 78.2 . The stories dealt with events on the Ohio University campus and in nearby Ohio cities. As had been the case in Experiment 1, each story contained two targets for the subsequent recognition test. One target in each story and its distractor bore no special relation to each other. In two stories, the other target and its distractor bore a synonymous relation to each other. In two other stories, the target and distractor bore an inferential relation to each other. An example of the synonymous pair is "But on the first pitch he hit the ball over the fence onto the Grover Center roof" (target) and "But on the tirst pitch he hit the ball over the fence onto the gym roof" (distractor). Ohio students, but not Oregon students, would know that the gym is Grover Center. The other synonym targetdistractor pair also used a building's proper and generic names. An example of a inferential pair is "The River Museum contains relics of steamboats that traveled up and down the river" (target) and "The River Museum contains relics of steamboats that traveled up and down the Ohio River" (distractor). Ohio, but not Oregon, students would have been able to infer that since the story described a museum in the nearby town of Marietta, the river in question must have been the Ohio River. The other inference target was a sentence describing an employee's drive of 75 miles from Athens to the state's main office complex. The inference distractor mentioned the state capital, Columbus, which Ohio students would know was 75 miles away from Athens.

Procedure. The procedure was the same as that used in Experiment 1, except that $22 \mathrm{~min}$ elapsed between the four stories and the recognition test, and arithmetic and contingency estimation tasks filled this interval. The same slide order and counterbalancing procedures used in Experiment 1 were used in Experiment 2.

\section{Results}

A 2 (slide order) $\times 2$ (knowledge) $\times 3$ (sentence type: synonym, inference, control) $\times 2$ (story) $\times 2$ (targetdistractor) analysis of variance was done on the recognition ratings. The predicted knowledge $\mathrm{x}$ target-distractor $\mathrm{x}$ sentence type interaction was again highly significant $[\mathrm{F}(2,56)=8.70, \mathrm{MSe}=4.97]$. Figure $2 \mathrm{a}$ depicts the recognition ratings for the control sentences. The usual benefit of expertise is present: The Ohio experts are more likely than the nonexperts to recognize the targets and reject the distractors. An analysis of variance on these control items alone resulted in a highly significant knowledge $\mathrm{x}$ target-distractor interaction consistent with this interpretation $[\mathrm{F}(1,28)=10.40, \mathrm{MSe}=3.79]$.

Figure $2 b$ depicts the recognition ratings for the inference sentences. Here the data are drastically different. The experts are less likely than the nonexperts to recognize the targets and reject the distractors. The analysis of variance on the inference sentences alone reveals the significant knowledge $\mathrm{x}$ target-distractor interaction $[\mathrm{F}(1,28)=6.42, \mathrm{MSe}=6.85] .^{5}$

Figure $2 \mathrm{c}$ depicts the data for the synonym sentences. The data in Figure 2c appear similar to the inference data in that experts appear to perform more poorly than 


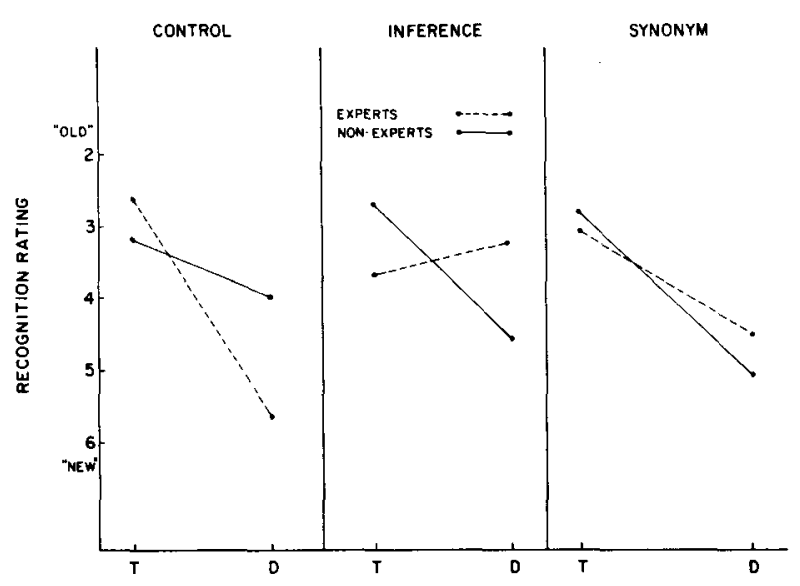

Figure 2. Left: Recognition of control sentences by experts and nonexperts. Center: Recognition of inference sentences. Right: Recognition of synonym sentences.

do the nonexperts. However, the knowledge $\mathrm{x}$ targetdistractor interaction was not significant. We hypothesized that this lack of significance may be at least in part accounted for by the marginally significant order $x$ story $\mathrm{x}$ knowledge $\mathrm{x}$ target-distractor interaction in the synonym data $[\mathrm{F}(1,28)=4.12, \mathrm{p}=.052, \mathrm{MSe}=4.18]$. When we examined the two orders of the recognition items (forward, reverse) and the two stories containing a synonym item, we found that in three of these four cases the nonexperts performed better than the experts did, just as we had predicted. However, when the "Grover Center" target preceded the "gym" distractor in the recognition test, the experts performed much better than the nonexperts did. We believe that this result occurred because when "Grover Center" occurred first, this distinctive target was given a very "old" rating (1.87) by the Ohio students. When the distractor later occurred, every Ohio "expert" gave it a "7," already having decided the earlier sentence was the target. Because the targets and distractors both occurred in a very short recognition test, once a subject rated one member of the pair, the rating of the other member of the pair was undoubtedly influenced. We think that this is the reason that the attractive option "Grover Center" precluded any false alarms on the subsequent distractor.

Despite the fact that no other such anomaly had been detected by higher order interactions, we examined individually each of the 24 tests of our hypotheses in the present two studies. The control sentences contained eight tests ( 2 experiments $\times 2$ slide orders $\times 2$ stories) in which the high-knowledge group was predicted to have better discrimination between targets and distractors than the low-knowledge group. All eight tests supported this prediction.

The synonym and inference sentences each contained 8 tests also. With the exception of one inference story in Experiment 1, the low-knowledge group was predicted to have better discrimination between targets and distractors than the high-knowledge group had, the opposite prediction from that in the case of the control sentences. Of those 16 tests of the hypotheses, 14 were supported. The "Grover Center" example cited above constituted one of the two violations. The other violation was an Experiment 1 inference sentence. In one slide order, the hypothesis was clearly supported. In the other slide order (target presented first), the hypothesis was narrowly disconfirmed. In summary, slide order made no difference in tests of the control sentences; it did compromise the results in two of the 16 synonym and inference sentences.

\section{Discussion}

Consider a finding reported by Chase and Simon (1973, p. 60). A chessmaster, a class A player, and a beginner are given trials consisting of 5 -sec exposures to a chessboard containing random placement of pieces from a middle game. Subjects are supposed to reconstruct the positions of the chess pieces from memory. The intriguing result was that the chessmaster performed the worst. We suspect that this "expert" was trying to "go beyond the information given" (Bruner, 1957). Having learned an immense amount of information from his prior experience, this expert attempted to place the pieces according to the strategies and rules he or she had learned. Since such rules are violated when pieces are arranged randomly, the chessmaster was at a disadvantage. Later in the article (p. 77), Chase and Simon (1973) suggested that the masters' typically superior memory performance for a legally arranged board is due to the fact that the master "will get more information from the partially reconstructed board than weaker players about what the rest of the position should be." As the master peruses the board during the reconstruction effort, the well-learned rules and strategies provide the expert with the tools to infer the remaining pieces. The weaker players cannot make such inferences.

We believe that an analogous result occurred in our studies. Our baseball and Ohio experts have the knowledge that enables them to go beyond the information given. As was the case with the chessmasters, this ability is typically an advantage. When the memory defeats this knowledge by voiding the rules of chess (Chase \& Simon, 1973) or by providing distractors whose presence can also be inferred from the prior information, then the prior knowledge is a liability.

The fact that experts were superior to nonexperts in their recognition of control sentences is consistent with prior research (e.g., Chiesi et al. 1979). The experts have a prior knowledge structure with which the targets could be interpreted and into which the targets were assimilated. The control distractors, unlike the other distractors, would not be assimilated, since they were neither a synonym nor an inference of presented material. Experts therefore were not at a disadvantage when the target and distractor were unrelated. When the 
target and distractor were related in a way apparent only to an expert, then the paradox of expertise existed. This paradox may contribute to the rampant overconfidence shown by experts in numerous real-world judgment tasks (Fischhoff, 1982, p. 439). Experts in such tasks are presented with stimuli and then asked to draw a conclusion, make a diagnosis, or render some other judgment. Experts, but not novices, are capable of inferring the presence of stimuli consistent with those actually presented (Arkes \& Harkness, 1980). Research by Johnson, Taylor, and Raye (1977) suggested that these self-generated stimuli and stimuli actually presented are capable of being confused. Thus, experts who infer the presence of stimuli consistent with the diagnosis or judgment would mistakenly believe that there existed more corroborating evidence. Confidence in their decision would thereby be unduly inflated. Of course, novices unable to generate the consistent stimuli would be basing their decision on a more veridical memory base. This would be another manifestation of the paradox of expertise.

\section{REFERENCES}

Andengon, J. R. Language, memory, and thought. Hillsdale, N.J: Erlbaum, 1976.

Anderson, J. R., Bower, G. H. Human associative memory. Washington, D.C: Hemisphere Press, 1973.

Arkes, H. R., \& Harkness, A. R. The effect of making a diagnosis on subsequent recognition of symptoms. Journal of Experimental Psychology: Human Learning and Memory, 1980, 6 , 568-575.

Bransford, J. D., Barclay, J. R., \& Franks, J. J. Sentence memory: A constructive versus interpretive approach. Cognitive Psychology, 1972, 3, 193-209.

Bruner, J. S. Going beyond the information given. In H. Gruber, K. Hammond, \& R. Jesser (Eds.), Contemporary approaches to education. Cambridge, Mass: Harvard University Press, 1957.

Charness, N. Components of skill in bridge. Canadian Journal of Psychology, 1979, 33, 1-50.

Chase, W. G., \& Simon, H. A. Perception in chess. Cognitive Psychology, 1973, 4, 55-81.

Chiesi, H. L., Spilich, G. J., \& Voss, J. F. Acquisition of domain-related information in relation to high and low domain knowledge. Journal of Verbal Learning and Verbal Behavior, 1979, 18, 257-274.

DEGroot, A. D. Thought and choice in chess. The Hague: Mouton, 1965.

Egan, D. E., \& Schwartz, B. J. Chunking in recall of symbolic diagrams. Memory \& Cognition, 1979, 7, 149-158.

Fischroff, B. Debiasing. In D. Kahneman, P. Slovic, \& A. Tversky (Eds.), Judgement under uncertainty: Heuristics and biases. Cambridge: Cambridge University Press, 1982.
FLESCH, R. F. A new readability yardstick. Journal of Applied Psychology, 1948, 32, 221-233.

HaRRIS, R. J. Inferences in information processing. In G. $\mathbf{H}$. Bower (Ed.), The psychology of learning and motivation (Vol. 15). New York: Academic Press, 1981.

Johnson, M. K., Taylor, T. H., Raye, C. Fact and fantasy: The effects of internally generated events on the apparent frequency of externally generated events. Memory Cognition, 1977, 5 , 116-122.

Martin, E. Transfer of verbal paired associates. Psychological Review, 1965, 72, 327-343.

Osooon, C. E. The similarity paradox in human learning: A resolution. Psychological Review, 1949, 56, 132-143.

REDER, L. M., \& ANDERson, J. R. A partial resolution of the paradox of interference: The role of integrating knowledge. Cognitive Psychology, 1980, 12, 447-472.

REDER, L. M., \& Ross, B. H. Integrated knowledge in different tasks: The role of retrieval strategy on fan effects. Journal of Experimental Psychology: Learning, Memory, and Cognition, 1983, 9, 55-72.

Reitman, J. S. Skilled perception in Go: Deducing memory structures from inter-response times. Cognitive Psychology, 1976, $8,336-356$.

Schank, R. C., \& Abelson, R. P. Scripts, plans, goals, and understanding. Hillsdale, N.J: Erlbaum, 1977.

Smith, E. E., Adams, N., \& Schorr, D. Fact retrieval and the paradex of interference. Cognitive Psychology, 1978, 10, 438-464.

Sulin, R. A., \& Dooling, D. J. Intrusion of a thematic idea in retention of prose. Journal of Experimental Psychology, 1974, 103, 255-262.

\section{NOTES}

1. We actually used 16 of the bottom 17 scorers. In order to have eight nonexperts in the forward slide order and eight in the backward slide order, we had to skip one forward-order nonexpert, who scored 4 , in order to obtain our eighth backwardorder nonexpert, who scored 5 .

2. There were two pairs of inference target-distractor recognition ratings and two pairs of synonym target-distractor recognition ratings. Since there were four pairs of control sentences (one per story), we averaged the ratings of the two pairs located in the inference stories and thus made one inference control pair. We did the same for the control sentences located in the synonym stories. We therefore analyzed two inference, two synonym, and two control target-distractor pairs.

3. We performed this analysis first with slide order as an independent variable. Since neither the main effect nor any of its interactions were significant, we collapsed over this variable.

4. The story main effect and the story $x$ target-distractor and story $\mathrm{x}$ order interactions were significant in this analysis. None is theoretically significant.

5. The order $\mathrm{x}$ story $\mathrm{x}$ target-distractor triple interaction was also significant. It is of no theoretical interest.

(Manuscript received February 1, 1983;

revision accepted for publication September 20,1983.) 